\title{
Two techniques for PIV-based aeroacoustic prediction and their application to a rod-airfoil experiment
}

\author{
Peter Moore • Valerio Lorenzoni • Fulvio Scarano
}

Received: 20 January 2010/Revised: 28 May 2010/ Accepted: 29 June 2010/Published online: 28 July 2010

(C) The Author(s) 2010. This article is published with open access at Springerlink.com

\begin{abstract}
In this paper, we discuss techniques by which the aeroacoustic properties of the turbulent flow in a rod-airfoil benchmark experiment can be inferred from time-resolved PIV measurement. While acoustic measurements can be made directly using microphones, the proposed techniques provide a means to directly link acoustic waves with specific flow events, which is invaluable in devising noise mitigation strategies. The approaches are possible thanks to recent improvements in digital and camera technology that can provide time-resolved measurements in air flows, necessary for the determination of unsteady flow quantities related to aeroacoustic production. Both techniques are based on Curle's acoustic analogy, where one is based on Lagrangian determination of the required quantities, while the other requires all flow quantities to be converted into Fourier modes. Application of both techniques yields results that are in reasonable agreement with microphone noise measurements for the rod-airfoil experiment.
\end{abstract}

\section{Introduction}

The abatement of aeroacoustic noise from land and air vehicles can be aided by new diagnostic techniques that directly link specific vehicle elements and local structures of the air flow around the vehicles with individual contributions to the emitted acoustic waves. In the present paper, we present techniques to apply aeroacoustic analogies directly to experimental time-resolved PIV data and evaluate these techniques for a rod-airfoil benchmark experiment.

P. Moore $(\varangle) \cdot$ V. Lorenzoni $\cdot$ F. Scarano Kluyverweg 2, 2629 Delft, HT, The Netherlands e-mail: p.d.moore@tudelft.nl
Acoustic diagnostics is usually done by microphone. Such techniques directly give the total acoustic emission intensity at the measured locations. Some source and contribution information is also available using techniques such as beam forming (see for instance Krim and Viberg 1996 for a review of early work) and two point correlations of the acoustic wave with a relevant flow quantity obtained by PIV say (such as in the work of Henning et al. 2008). However, these techniques give only limited information about the connection between the acoustic source and the turbulent flow. Beam forming techniques localize individual source contributions but do not give extended information of the nature of the individual sources. Two point correlations can give similar information, but the information is limited by the choice of flow quantity for the correlation, which may or may not be well related to the actual acoustic source.

In numerical studies at low speeds, acoustic predictions are often performed by the time resolved simulation of a flow and post-priori use of a suitable aeroacoustic analogy (see Wang et al. 2006 for a recent review). In this way, the acoustic emissions are decoupled from the purely hydrodynamic flow simulation. Typically, these approaches require high fidelity simulations, while the acoustic analogy implementations must be carefully implemented with robustness in mind (see for instance Martinez-Lera et al. 2008). Moreover, these simulations are usually computationally expensive (see Wang et al. 2006 for a discussion), while cheaper simulation techniques such as RANS-based approaches are generally unsuitable for aeroacoustics problems. Further, simulation around complex geometries is difficult, and the degree of modeling required to include even idealized geometries can lead to a loss of confidence in the accuracy of the solutions, particularly for design purposes.

The techniques we have been developing combine timeresolved PIV for flow information with acoustic analogies 
for detailed acoustic analysis. These techniques are especially suited for flows around complex geometries, which are expensive to investigate numerically, and in any case, usually require idealization of the geometries. These techniques are only recently possible for air flows, with the development of rapid-rate camera systems for high speed acquisition.

The techniques developed are applied to a time-resolved rod-airfoil PIV experiment, which is a useful benchmark for turbulence structure interaction noise (Greschner et al. 2008; Jacob et al. 2005) and has received quite some treatment including Jacob et al. (2008), Siller et al. (2005), and Casalino et al. (2003). This benchmark is particularly tractable for aeroacoustic analysis, as the dominant acoustic source is the impingement of large 2-D vortex rolls on the airfoil leading edge, which are readily resolvable by the PIV measurement.

The direct computation of sound from PIV flow measurements has already been achieved for certain flow configurations. For example, Schram et al. (2007) have investigated the noise associated with axisymmetric vortex pairing in round jet flows. This is achieved by the application of Möhring's analogy (Moehring 1978). Schram et al. (2007) note that formulations that reinforce physical conservation laws can lead to more robust predictions with regard to experimental error for this kind of flow. Recently, Haigermoser (2009) has applied Curle's acoustic analogy to PIV cavity data in water, while Koschatzky et al. (2009, 2010) has done similarly in air. Other uses of PIV data in aeroacoustics has typically involved identifying flow-acoustic correlations, such as Schröder et al. (2004), Wernet (2007), which can lead to an heuristic understanding of the link between certain flow features and the acoustic emissions.

In this paper, we present two techniques for the coupling of PIV-experimental data with an acoustic analogy in an air flow around a rod-airfoil configuration. In particular, we pay attention to the constraints placed upon the data by the nature of the experimental data itself, especially limits in temporal resolution and measurement uncertainty. The methods and limitations are demonstrated by application to a two-dimensional time-resolved PIV experiment of the rod-airfoil configuration.

\section{Experimental setup}

Combined PIV and acoustic measurements were previously undertaken on the flow around an airfoil in the wake of a cylinder, both placed in an anechoic wind tunnel (Lorenzoni et al. 2009). Relevant details of these experiments are repeated here.

The PIV measurements were performed with an acquisition rate of $2,700 \mathrm{~Hz}$, giving a time-resolved planar field of view around a NACA0012 airfoil with a chord of $100 \mathrm{~mm}$. The airfoil was located $220 \mathrm{~mm}$ downstream in the wake of a 6-mm-diameter rod at zero degrees angle of attack, and both were placed in a wind tunnel operating at $15 \mathrm{~m} / \mathrm{s}$ with exit dimensions of $0.51 \times 0.38 \mathrm{~m}^{2}$. The rod periodically sheds Kármán vortices at a frequency of approximately $500 \mathrm{~Hz}$, which interact with the downstream airfoil producing a highly tonal noise of the same frequency, which is captured by microphones. The Reynolds number of the flow is 6,000 based on the diameter of the rod and 100,000 based on the airfoil chord. The main details are illustrated in Fig. 1.

Two-component time-resolved particle image velocimetry (TR-PIV) was used to obtain planar velocity field measurements. Two Photron Fast CAM SA1 CMOS cameras $(1,024 \times 1,024$ pixels, $20 \mu \mathrm{m}$ pixel pitch) were combined to image the flow around the entire airfoil. The flow was seeded with smoke particles (approximately $1 \mu \mathrm{m}$ diameter). The combined field of view of both cameras (field of view B in Fig. 1) corresponded to $164 \times 83 \mathrm{~mm}^{2}$ and $1,939 \times 1,024$ pixels, and 2,700 image pairs were obtained.

The velocity fields were evaluated from the image recordings using the Window Deformation Iterative Multigrid algorithm of Scarano and Riethmuller (1999). The interrogation window for the correlation corresponded to $21 \times 21$ pixels with a $75 \%$ overlap. This gave a spatial resolution of the velocity field corresponding to 2.5 vectors/ $\mathrm{mm}$. The PIV measurements include the actual airfoil surface, but the correlated images were only accurate from the third vector from the airfoil surface, at just over $1 \mathrm{~mm}$.

Acoustic measurements were simultaneously obtained at four locations at a radius of $1.25 \mathrm{~m}$ from the airfoil leading edge. These locations were put at angles with respect to the airfoil chord of $90^{\circ}, 117^{\circ}, 135^{\circ}, 143^{\circ}$. The microphones were LinearX-M51, omnidirectional pressure microphones that acquired for $20 \mathrm{~s}$ with a sampling frequency of $51.2 \mathrm{kHz}$ and had a frequency resolution of $12.5 \mathrm{~Hz}$.

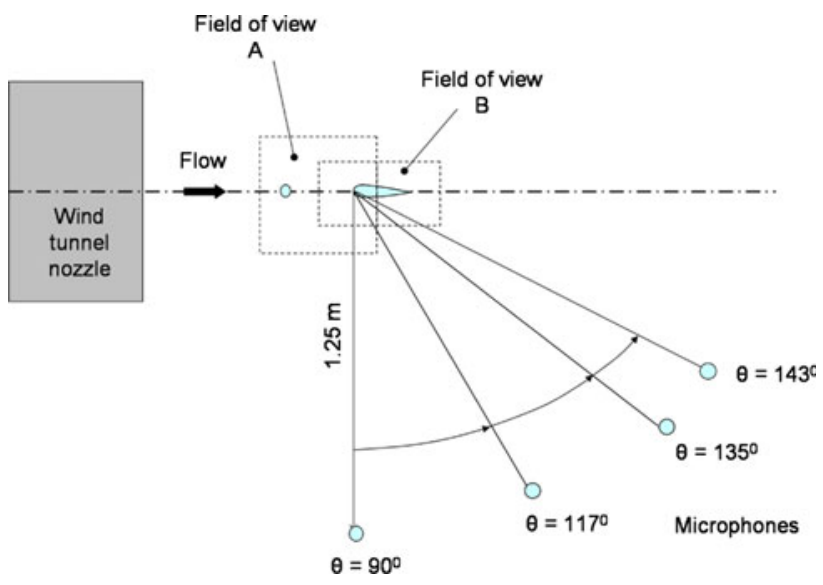

Fig. 1 The main features of the experimental setup (top view) 


\section{Theory}

The hybrid computational aeroacoustic approach typically involves the simulation of flow features governed by the incompressible Navier-Stokes equations, and the use of an acoustic analogy to add the compressible part of the flow, due to acoustic waves, in a secondary step. The essential assumption of this approach, that the production and propagation of acoustic waves can be decoupled from the flow, has been found to be quite reasonable approach if the Mach number is small enough. One such acoustic analogy, specifically concerned with flows around stationary bodies, is Curle's acoustic analogy (Curle 1955). For the far-field sound emitted by a compact subsonic body, this can be written as

$P(\mathbf{x}, y)=\frac{1}{4 \pi a_{o}} \frac{x_{i}}{r^{2}} \frac{\partial}{\partial t} \int_{S}-l_{j} \delta_{i j} p\left(\mathbf{y}, t-\frac{r}{a_{0}}\right) \mathrm{d} S(\mathbf{y})+Q$,

where $P$ is the acoustic pressure prediction at the far-field location, $p$ is the static pressure on the emitting body surface, $\mathbf{x}$ and time $t, S$ is the surface of the body, $y$ are integration points on the body surface, $a_{0}$ is the speed of sound, $r$ is the distance between an observer in the far field and a representative origin location, $l_{j}$ is normal to the airfoil, $\delta_{i j}$ is the Kronecker-delta function and $Q$ represents additional noise effects that will be neglected (for example due to turbulent mixing, or viscous attenuation of acoustic waves). Note that the chosen form for equation (1) explicitly lumps a volume integral within $Q$ relating to the quadrupole noise emission of free turbulence. This can be demonstrated to have negligible impact in the low Mach number limit (Curle 1955). Viscous attenuation of acoustic sources depends on the distance of wave propagation and is similarly negligible for the distances considered in this paper.

To evaluate equation (1), knowledge of the time derivative of the surface pressure variation on the solid structure embedded in the flow is required. Importantly, it is only required that the pressure is known accurately enough to resolve static pressure amplitudes at the frequencies of interest, resolution of amplitudes due to acoustic waves is not required. Directly resolving acoustic wave amplitudes would be incredibly difficult, considering that these are many orders of magnitude smaller than those of the turbulent flow.

For the case of an airfoil acting as a compact source, evaluation of equation (1) is equivalent to evaluating the fluctuating lift on the airfoil (Curle 1955; Howe 2001). Since this information is most often given directly by CFD solvers, the question this paper is concerned with is, can this information also be obtained with sufficient accuracy by time-resolved PIV? Such a combination would be attractive as an additional experimental technique for noise prediction (without the need of microphones) and noise source determination, as clear understanding of the link between the development of flow structures and the emitted noise can lead to new passive or active control strategies for noise mitigation. In this paper, we propose and evaluate two candidate techniques for noise prediction based on time-resolved PIV data.

\section{Acoustic prediction in the time domain}

We use a multistep procedure that leads from the acquisition of time-resolved PIV data to the prediction of aeroacoustic emission. The steps are the following:

1. Time-resolved acquisition of the particle images and correlation for velocity fields using PIV.

2. Evaluation of the terms in the Navier-Stokes equations from the PIV data.

3. Solution of a Poisson equation formed from the Navier-Stokes equations to obtain the static pressure field.

4. Integration of Curle's acoustic analogy to obtain the far-field pressure.

Essentially, to complete these steps, following the works of Liu and Katz (2006) and de Kat et al. (2008), the NavierStokes equations are rearranged to the following form:

$\nabla p=\rho\left(-\frac{\mathrm{D} \mathbf{u}}{\mathrm{D} t}+v \nabla^{2} \mathbf{u}\right)$,

where $v$ is kinematic viscosity, $\mathbf{u}(\mathbf{x}, t)$ is the flow velocity at position $\mathbf{x}$ and time $t, \rho$ is the density and $p$ is the fluid pressure. For step 2, ignoring the effects of viscosity, we determine the material derivative of the velocity, or material acceleration, $\frac{\mathrm{Du}}{\mathrm{D} t}$, given by

$\frac{\mathrm{D} \mathbf{u}}{\mathrm{D} t}=\frac{\partial \mathbf{u}}{\partial t}+(\mathbf{u} \cdot \nabla) \mathbf{u}$.

Step 3 involves solving equation (2) for the static pressure on the left-hand side, while the final step, 4 , is to solve Curle's acoustic analogy in equation (1) to obtain the farfield pressure due to acoustic waves from the near-field static pressure distribution. Important aspects of these steps are now explained.

4.1 Time-resolved acquisition of the particle images and correlation for velocity fields using PIV

The first step in this technique involves the acquisition of particle image fields and their processing to obtain velocity fields. Two important points should be observed to enable acoustic predictions. First, the acquisition should be time 
resolved. A description of what is required by time resolved is found in the following sections. Secondly, the entire noise producing region should be encompassed within the experiment and preferably the entire noise producing body. Details for the experiment used to demonstrate the techniques in this paper are given in Sect. 2, as well as by Lorenzoni et al. (2009).

\subsection{Evaluation of the material acceleration}

The evaluation of the material acceleration, $\frac{\mathrm{Du}}{\mathrm{D} t}$, from the PIV velocity fields is the first step required downstream of the experimental acquisition of the velocity fields. The calculation of derivatives in particular from PIV velocity data must be carefully evaluated. For the case of calculation of derivatives using finite difference approximations, there are two distinct sources of uncertainty that should be considered. First, there is the Taylor round-off uncertainty associated with using a finite scheme to approximate an instantaneous flow characteristic. The order of this uncertainty is related to the order of the Taylor scheme used and grows with increasing measurement step (either in space or time). The second important source of uncertainty is directly introduced by measurement uncertainty. For uncorrelated measurements, this uncertainty becomes large as the measurement step decreases in size, because in this limit, the uncorrelated uncertainty difference does not change order of size and dominates the increasingly smaller difference that is to be measured. This source of uncertainty is particularly important for temporal derivatives, as there is no correlation in time of the measurement uncertainty. For the computation of the material acceleration, in the following two sections we outline what we mean by Eulerian evaluation and Lagrangian evaluation.

An analysis of the role of time step on both kinds of aforementioned errors is undertaken by Violato et al. (2010) (a condensed version of this paper is given by Violato et al. 2009) using a 3-D Tomographic PIV investigation into the same geometry as the experiment described here, but in this case the Kármán vortices were shed at $100 \mathrm{~Hz}$, while the acquisition frequency was $5,000 \mathrm{~Hz}$ and the Reynolds number based on the cylinder chord was 3,500 , so that the Kármán vortices were sampled at 50 times per cycle, in place of the 5.4 times achieved in the experiment this paper is based on. These errors were investigated for Eulerian and Lagrangian evaluations using the methods described in the following sections. Violato et al. (2010) find that at short time separations $(0.4 \mathrm{~ms}$ or $4 \%$ of the Kármán shedding frequency), both Eulerian and Lagrangian calculations yield almost results for the material derivative, but both are affected by measurement uncertainty leading to large nonphysical fluctuations in the computed values. At larger time separations, the predictions of the Eulerian and Lagrangian techniques diverge as the Eulerian calculations are found to quickly suffer Taylor round-off error. The result is not surprising when one considers that in largely convected flow regions, the timescale of point-wise Eulerian variations is considerably shorter than that of variations along Lagrangian trajectories (Tennekes 1975; Koeltzsch 1999). The optimal time separation along a Lagrangian trajectory was identified as $1.4 \mathrm{~ms}$, or $14 \%$ of the shedding period.

Moreover, Violato et al. (2010) investigated one further issue of criticality for Lagrangian determinations. For 2-D measurements, the following methods use a projection of the 3-D path into the 2-D measurement plane. Violato et al. (2010) demonstrated that this projection did not significantly change the computed in-plane material acceleration or the resultant computed pressure.

\subsubsection{Eulerian evaluation of the material acceleration}

For the experimental data analyzed in this paper, the acquisition frequency (at 5 times the rod shedding frequency) was significant enough to temporally resolve structures as they evolved, but only in the Lagrangian sense of following the flow motion. For the calculations of the material acceleration of the flow, $\frac{\mathrm{Du}}{\mathrm{D} t}$, the most obvious method to attempt at the outset is to use equation (3) to separate the material acceleration into its Eulerian components. The determination of the material acceleration is then equivalent to the separate evaluation of the Eulerian acceleration and the convective derivative. However, the direct determination of the Eulerian acceleration $\frac{\partial \mathbf{u}}{\partial t}$ on the right-hand side of this equation by finite difference method such as

$\frac{\mathrm{d} \mathbf{u}_{n}}{\mathrm{~d} t} \approx \frac{\mathbf{u}_{n+1}-\mathbf{u}_{n-1}}{2 \mathrm{~d} t}$

was found by Violato et al. (2010) to be only valid over much shorter time separations than those resolved in the experiment used for this paper and at those short time separations, to be affected by measurement uncertainty leading to misleading results for the material acceleration $\frac{\mathrm{Du}}{\mathrm{D} t}$ See the previous section for a discussion.

\subsubsection{Lagrangian evaluation of the material acceleration}

For flows which are predominantly convected, Lagrangian timescales are much longer than Eulerian timescales (see for instance Koeltzsch 1999 for a discussion). In this case, higher temporal resolution is achievable by computing flow quantities in a Lagrangian manner. From a Lagrangian point of view, the material acceleration is simply the acceleration of parcels of flow along fluid pathlines. If the pathlines can be determined with sufficient accuracy, then $\frac{\mathrm{Du}}{\mathrm{D} t}$ can be evaluated, again by a finite difference technique 
say, along the pathline. The chief advantage of this approach is that the time resolution of the PIV system is sufficient to capture the development of flow features, in a reference frame that is moving with those features; however, it is not sufficient to capture the point-wise instantaneous changes of an Eulerian perspective.

Liu and Katz (2006) first presented a method to compute the Lagrangian acceleration from a PIV acquisition. While their method gave some inspiration, our approach proceeds on a somewhat different line, which is now outlined.

Suppose first that we have a complete description of the flow velocity field in space and time given by $\mathbf{u}(\mathbf{x}, t)$. Then, the fluid path location $\mathbf{x}_{b}$ at time $t_{b}$ which evolved from the location $\mathbf{x}_{a}$ at time $t_{a}$ is given by

$\mathbf{x}_{b}=\mathbf{x}_{a}+\int_{t_{a}}^{t_{b}} \mathbf{u}(\mathbf{l}(t), t) \mathrm{d} t$,

where $\mathbf{l}(t)$ is the instantaneous position on a path from $\mathbf{x}_{a}$ to $\mathbf{x}_{b}$ such that along the path $\mathbf{l}(t)$, the instantaneous velocity, $\mathbf{u}$, is tangential to the path itself. That is

$\mathbf{u}(\mathbf{l}(t), t) \cdot \mathbf{l}(t)=0$

The problem becomes one of estimating the pathlines. The main difficulties to do this are that the velocities are only available at discrete time steps, measurement uncertainty and that only 2-D measurements of the flow are available. The method we propose is to first introduce a set of intermediate velocity fields between measured velocity fields, using a linear interpolation of the measured velocity fields. That is, supposing at time step $n$, a set of velocity fields $\left\{\mathbf{u}_{n}\right\}$ is measured, and at time step $n+1$, a set of velocity fields $\left\{\mathbf{u}_{n+1}\right\}$ is measured throughout a domain $D$. Then, we form $M-1$ sets of intermediate velocity fields given by

$\mathbf{u}_{n, m}=\mathbf{u}_{n}+\frac{m}{M}\left(\mathbf{u}_{n+1}-\mathbf{u}_{n}\right), \quad m=1 \ldots M-1$,

while $\mathbf{u}_{n, 0}=\mathbf{u}_{n}$ and $\mathbf{u}_{n, m}=\mathbf{u}_{n+1}$. The pathlines are then approximated by linear projections from each of the subintervals, such that the local subinterval velocity vector is parallel to the path, so that

$\mathbf{x}_{n+1}=\mathbf{x}_{n}+\sum_{m=0}^{M-1} \frac{\mathbf{u}_{n, m} \mathrm{~d} t}{M}$

for each $\mathbf{x}_{n}$ in $D$.

The value of $M$ can be made arbitrarily large, although eventually there will be meaningful gain to the results. For the analysis of this experiment, a value of $M=30$ was chosen, although in testing the results converged at a value of $M=10$. In general, this would be dependent on the details of the experiment in question. In this way, a path can be created that is reasonably smooth and can deal well with some intermediate curvature (for example due to shear gradients near a solid surface). The derivative itself is computed only with measured PIV fields, the interpolation is used only to allow for a good approximation of the pathline.

Note that in the case that $M=2$ (so that there is one intermediate velocity field), then the method reverts to that of Liu and Katz (2006), although retaining the benefit of being an explicit formulation. A comparison of the pathlines with and without the subinterval procedure is shown in Fig. 2. Significant deviation of the path is observable after one measurement interval.

\subsection{Solution of Navier-Stokes equation for static pressure}

To obtain the pressure $p$, equation (2) could be solved directly from a single reference point in the domain. However, because PIV measurements have some uncertainty, the constitutive equations in this case are not exactly representative of the measured data. Moreover, a 2-D description of the flow field is incomplete. While, as pointed out by Haigermoser (2009), pressure is a scalar and can be integrated in a plane without requiring the normal pressure gradients of equation (2), the projection of the material acceleration onto a 2-D plane is still an approximation that is only exactly valid in a perfectly 2 -D flow. Because of this, direct integration of equation (2) suffers from path-dependency problems. The method proposed by Liu and Katz (2006) is essentially to integrate the equation along all possible paths and to average the result. Alternatively, here, we follow the approach of de Kat et al. (2008) in forming a Poisson equation by taking the divergence of equation (2).

$\nabla^{2} p=\rho \nabla \cdot\left(-\frac{\mathrm{D} \mathbf{u}}{\mathrm{D} t}+v \nabla^{2} \mathbf{u}\right)$

This equation is solved with a standard Poisson solver, from suitable reference points for Dirichlet boundary conditions and using the pressure gradients given by equation (2) for

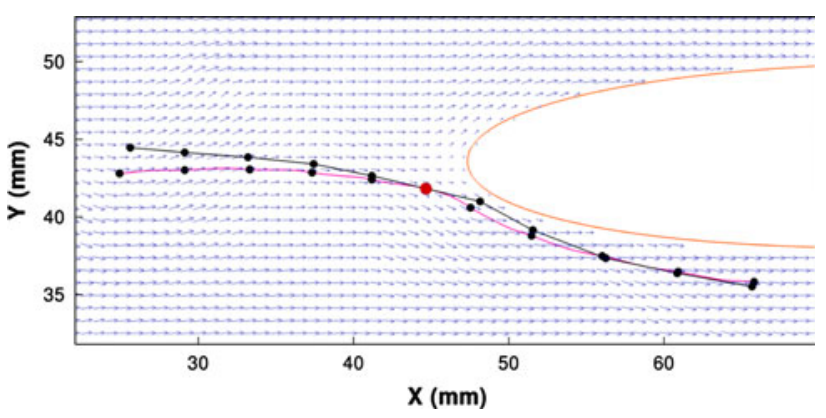

Fig. 2 Lagrangian pathlines forward and backward in time from a common ( $r e d$ ) point with (every second) velocity vector at the time step of the path center. Black pathline is $M=1$ and pink pathline is $M=30$. Black points indicate times on the pathline at which a PIV measurement is available 
boundary conditions elsewhere as Neumann boundary conditions. Similarly, supposing equation (2) can be solved for the pressure $p$, then the time derivative of the pressure could also be obtained by finite difference. However, this may lead to inaccuracies when the time resolution of the data is not high. Instead, we propose that the time derivative of the pressure can also be obtained by first evaluating the material derivative of the pressure along the same pathlines as the material derivative is evaluated and then applying

$\frac{\partial p}{\partial t}=\frac{\mathrm{D} p}{\mathrm{D} t}-\mathbf{u} \cdot \nabla p$.

In evaluating this equation, $\nabla p$ is determined by taking the gradient of the solution of equation (2), rather than by directly using equation (2) itself.

Note that this method is used by Koschatzky et al. $(2009,2010)$ for the case of shear-layer-driven cavity flow, but in this case, flush-mounted microphones were placed in the cavity verifying that the static pressure is accurately computed from PIV using this method.

\subsection{Solution of Curle's acoustic analogy}

The solution of Curle's acoustic analogy in equation (1) is now rather straight forward. The integral is computed on a surface as close as possible to the physical airfoil surface (although not exactly on the surface due to PIV limitations). For this case of vortex interaction with an acoustically compact body, the noise is proportional to the fluctuating lift (Curle 1955; Howe 2001) generated by the deformation of large impinging vortices. With the integral surface much closer to the physical surface than the length scale of the impinging vortices, the fluctuating lift due to these deformations is still captured.

As the data are only available on a 2-D cross-section of the airfoil, the result is multiplied by the airfoil length to account for the entire airfoil contribution to the acoustic far field. The essential assumption is that the impinging vortex street is largely coherent over the airfoil length (this has been examined in Lorenzoni et al. 2009).

Acoustic predictions were made and compared to the microphone data at the $90^{\circ}$ and $143^{\circ}$ locations, both situated at a distance of $1.25 \mathrm{~m}$ from the airfoil leading edge. For this experiment, the frequency of the peak acoustic emissions was $500 \mathrm{~Hz}$, with a corresponding sound wavelength of $\lambda=0.7 \mathrm{~m}$. The far-field approximation of Curle's acoustic analogy is typically valid at least 1-2 wavelengths from the acoustic source, so direct comparison is valid for frequencies up to the peak. Moreover, the airfoil chord of $0.1 \mathrm{~m}$ is compact compared to the dominant sound wavelength of $\lambda=0.7 \mathrm{~m}$, although may not be at frequencies in the spectrum that are significantly higher.

\section{Acoustic prediction in the frequency domain}

An alternate method is to rewrite the equations using Fourier modes, especially since the phenomenon under investigation is periodic. Curle's acoustic analogy, equation (1), can be rewritten as

$\hat{P}_{n}(\mathbf{x})=\frac{1}{4 \pi} \int_{S} \frac{\partial}{\partial x_{i}}\left[e^{-2 \frac{\pi i n r}{a_{0}} T}\right] l_{j} \boldsymbol{\delta}_{i j} \hat{p}_{n} \mathrm{~d} S(\mathbf{y})$

where

$\hat{p}_{n}=\int_{-T / 2}^{T / 2} e^{-2 \frac{\pi i n t}{T}} p \mathrm{~d} t$

is the $n$-th mode of the Fourier transform of the pressure signal $p$ (independently at each spatial location $\mathbf{x}$ ), $T$ is the total measurement period and

$r=|\mathbf{x}-\mathbf{y}|$

is the distance between the observer location $\mathbf{x}$ and the surface integration point $\mathbf{y}$. To evaluate the Fourier modes of the pressure, instead of using the solution of equation (2) in equation (12), instead, we apply a Fourier transform to equation (2) and solve for each Fourier mode directly (again, for the purposes of this paper ignoring the viscous term). This approach is contained in equation (14)

$\nabla \hat{p}_{n}=-\rho\left(i \omega_{n} \hat{\mathbf{u}}_{n}+\mathcal{F}_{n}\left\{(\mathbf{u} \cdot \nabla) \mathbf{u}-v \nabla^{2} \mathbf{u}\right\}\right)$.

where $N$ is the total number of (odd) modes and

$\omega_{n}=\frac{2 \pi}{T} \begin{cases}n & n=0 \ldots(N-1) / 2 ; \\ (n-N), & n=(N+1) / 2 \ldots N-1 .\end{cases}$

\section{Results}

In the theory section, we alluded to the requirement for a Lagrangian evaluation of the material acceleration. Consider in Fig. 3 the solution of equation (3) in time, at the representative point of $(40.6,44.3 \mathrm{~mm})$. The material derivative, $\frac{\mathrm{D} u}{\mathrm{D} t}$ is negative, because flow is slowing as it approaches the leading edge of the airfoil. Interestingly, the Eulerian acceleration $\frac{\mathrm{d} u}{\mathrm{~d} t}$ (obtained from the difference of the material acceleration and the convective acceleration) is nearly canceling with the flow convective acceleration $\mathbf{u} \cdot \nabla u$.

This is important, because if the calculation was to proceed by directly calculating the Eulerian acceleration and the flow convective acceleration independently, small errors in the calculation of either can easily dominate the sum of the two. For the Eulerian acceleration, this is especially problematic, because the random noise of the measurements that is not correlating from one measurement to 


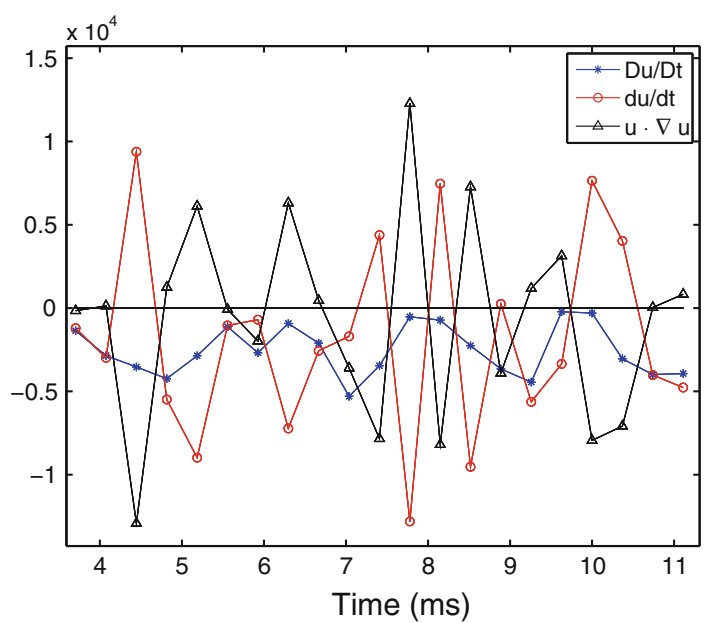

Fig. 3 Calculation of the material acceleration at the point (40.6, $44.3 \mathrm{~mm}$ )

the next leads to uncertainty in time derivatives, while insufficient temporal resolution leads to a significant Taylor truncation error.

In this section, we give some results of the application of both techniques to the experiment described in the experimental section. The first step of the Lagrangian approach involves the computation of the static pressure by solution of equation (9).

In Figs. 4 and 5, we show a typical result of such a computation along with vectors of the velocity, split into the mean and fluctuating components. In Fig. 4, the lowpressure zones corresponding to the flow acceleration above and below the airfoil are clearly evident, as well as the high-pressure region at the stagnation point at the airfoil leading edge. In Fig. 5, the vortex centers are clearly identifiable as regions of low pressure.

In Figs. 6 and 7, we compare the spectra of the acoustic field at two locations at $1.25 \mathrm{~m}$ from the airfoil leading edge. The peak height, peak frequency and trend with angle are certainly well captured by both methods. Both methods overestimate the high-frequency component of the signal, although the Fourier method more so. In principle, with a sampling frequency of $2,700 \mathrm{~Hz}$, the spectra should not be aliased up to a frequency of $1,350 \mathrm{~Hz}$. Our explanation for the overestimation is related to the 2-D assumption that is made. Essentially, we assume that the airfoil effectively radiates in phase along the span, as the motion is predominantly 2-D. In practice, especially at smaller scales and higher frequencies, the loss of span-wise coherence would lead to canceling interference of acoustic emissions and lower acoustic emission.

Finally in Fig. 8, we show contours of the acoustic pressure obtained by solution of equation (1). Acoustic waves propagate predominantly in directions normal to the

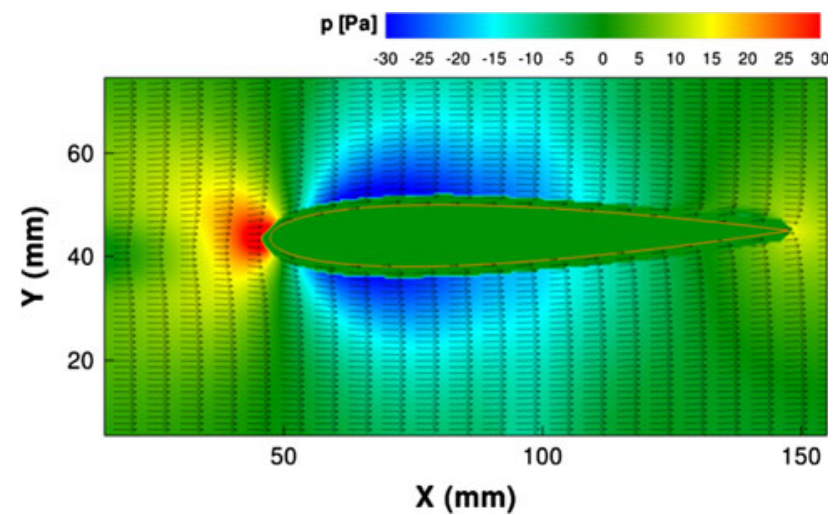

Fig. 4 Contours of the local mean pressure $\bar{p}$ with vectors of the local mean velocity $\overline{\mathbf{u}}$. Every third PIV vector is shown vertically, and every 15 th vector horizontally

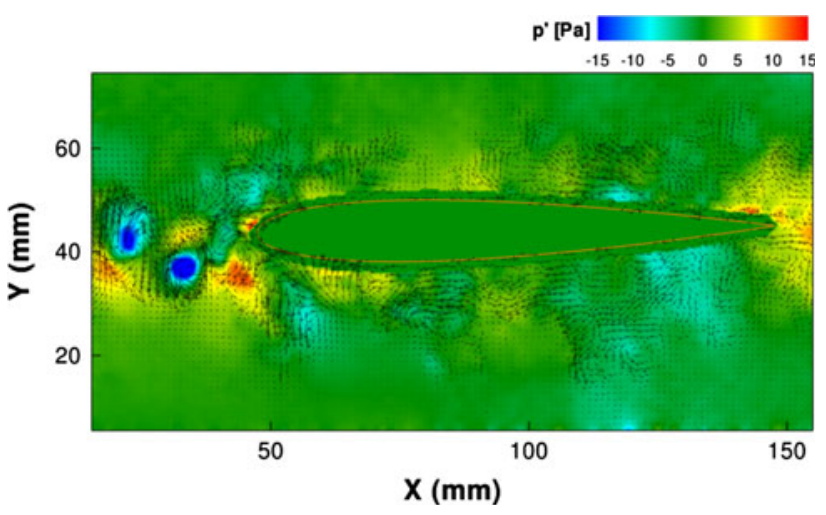

Fig. 5 Contours of the pressure perturbation $p^{\prime}=p-\bar{p}$ with vectors of the velocity perturbation $\mathbf{u}^{\prime}=\mathbf{u}-\overline{\mathbf{u}}$. Every third PIV vector is shown in each dimension

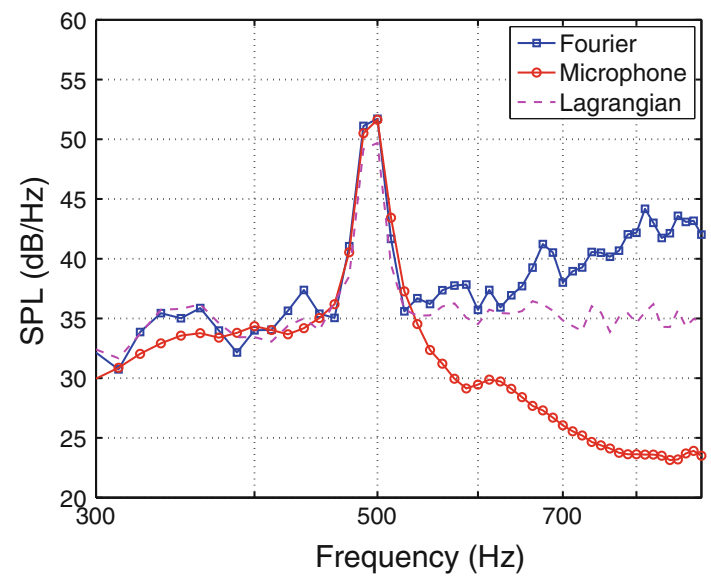

Fig. 6 Spectra comparison at $90^{\circ}$ between microphone measurements and Lagrangian and Fourier method

flow axis, and slightly upstream. This indicated directivity pattern is qualitatively the same as that identified by Jacob et al. (2005). 


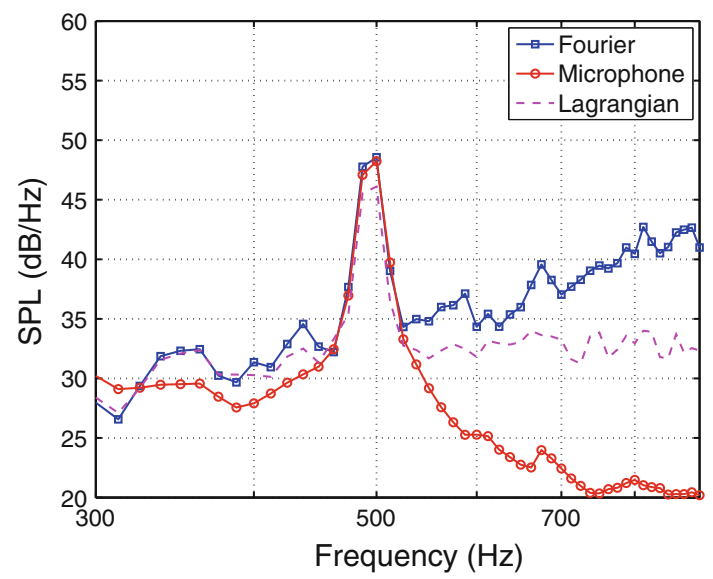

Fig. 7 Spectra comparison at $143^{\circ}$ between microphone measurements and Lagrangian and Fourier method

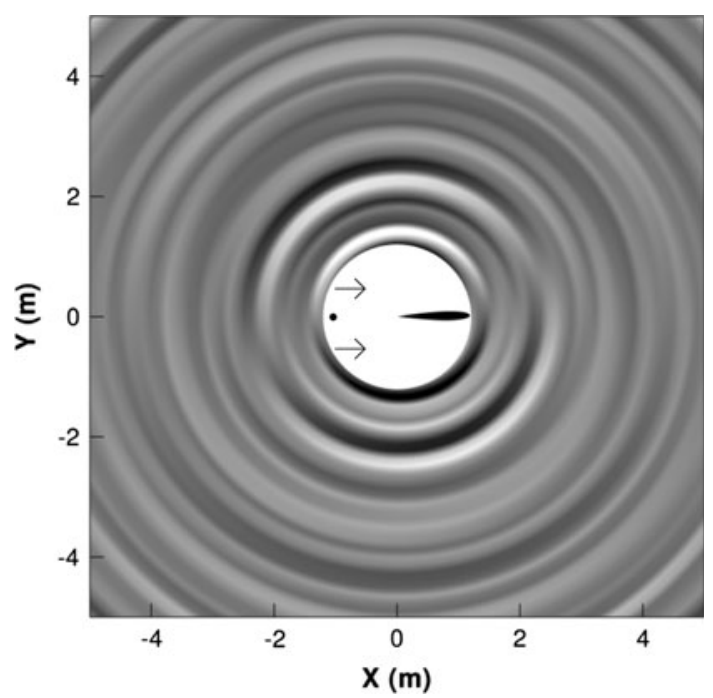

Fig. 8 Contours of the far-field pressure due to acoustic prediction. The rod and airfoil are indicated (but not to scale) as well as the flow direction

\section{Conclusions}

Two methods have been presented for evaluation of the farfield acoustic pressure with PIV flow data and applied to a rod-airfoil benchmark experiment. These methods are designed to avoid the direct computation of time derivatives of flow quantities, which is problematic due to both random noise in the PIV signals and limited temporal resolution. Indeed, it is shown that for the calculation of the material acceleration, even a small error in calculating the velocity time derivative can lead to an error that overwhelms the computation, due to the (nearly) canceling nature of the convection and derivative terms.

The acoustic spectra predicted by the two techniques show good agreement with the microphone measurements, display the correct directivity trend, and capture accurately the peak frequency, with the Fourier method giving almost identical peak heights to the microphone measurements and the Lagrangian method systematically around $2 \mathrm{~dB} / \mathrm{Hz}$ lower than the microphone measurements. This capture of the peak frequency is thus well predicted by both methods, although this is unsurprising given the tonal quality of the noise produced by the configuration. At frequencies higher than the peak, both methods diverge from the microphone data, despite the PIV measurements not being aliased at these frequencies. Some of this divergence may be explained as a result of the 2-D assumption with regard to coherent acoustic emission, where in practice particularly at higher frequencies, the loss of span-wise coherence would lead to canceling interference of acoustic emissions and lower acoustic emission.

In principle, the results of this paper do not really distinguish the two methods, as both fail to predict the highfrequency trend. While in principle we feel that a Lagrangian-type approach is more suitable for such convected flows, both methods appear suitable for the study of this, and related, problems of low-speed-flow compactbody interaction.

Finally, these techniques are applied specifically to the case of vortex interactions with an acoustically compact geometry. The extension of these techniques to more general flows (especially those in which the turbulent boundary layer is significant) is a challenge for the future.

Acknowledgments This work was conducted as part of the FLOVIST project (2008-2013), funded by the European Research Council, European Commission "Ideas" FP7 Programme. The authors also acknowledge the contribution of Roeland de Kat in providing a Poisson solver implementation. The authors also acknowledge Marthijn Tuinstra of the Dutch NLR as one of the providers of the experimental data used in this paper.

Open Access This article is distributed under the terms of the Creative Commons Attribution Noncommercial License which permits any noncommercial use, distribution, and reproduction in any medium, provided the original author(s) and source are credited.

\section{References}

Casalino D, Jacob M, Roger M (2003) Prediction of rod-airfoil interaction noise using the Ffowcs-Williams-Hawkings analogy. AIAA 41(2):182-191

Curle N (1955) The influence of solid boundaries upon aerodynamic sound. Proc Roy Soc Lon A 231:505-514

de Kat R, Oudheusden B, Scarano F (2008) Instantaneous planar pressure field determination around a square-section cylinder based on time-resolved stereo piv. In: 14th International symposium on the application of laser techniques to fluid mechanics, Lisbon, Portugal

Greschner B, Thiele F, Jacob M, Casalino D (2008) Prediction of sound generated by a rod-airfoil configuration using EASM DES 
and the generalised Lighthill/FW-H analogy. Comput Fluids $37: 402-413$

Haigermoser C (2009) Application of an acoustic analogy to PIV data from rectangular cavity flows. Exp Fluids

Henning A, Kaepernick K, Ehrenfried K, Koop L, Dillmann A (2008) Investigation of aeroacoustic noise generation by simultaneous particle image velocimetry and microphone measurements. Exp Fluids 45(6): 1073-1085

Howe M (2001) Unsteady lift and sound produced by an airfoil in a turbulent boundary layer. J Fluids Struct 15:207-225

Jacob M, Boudet J, Casalino D, Michard M (2005) A rod-airfoil experiment as benchmark for broadband noise modeling. Theor Comput Fluid Dyn 19:171-196

Jacob M, Ciardi M, Gamet L, Greschner B, Moon Y, Vallet I (2008) Assessment of CFD broadband noise predictions on a rod-airfoil benchmark computation. In: 14th AIAA/CEAS aeroacoustics conference 2008-2899

Koeltzsch K (1999) On the relationship between the lagrangian and eulerian time scale. Atmos Environ 33(1):117-128

Koschatzky V, Boersma BJ, Scarano F, Westerweel J (2009) High speed PIV applied to aerodynamic noise investigation. In: 8th International symposium on particle image velocimetry, Melbourne, Australia

Koschatzky V, Moore PD, Boersma BJ, Scarano F, Westerweel J (2010) High speed PIV applied to aerodynamic noise investigation. Submitted to experiments in fluids

Krim H, Viberg M (1996) Two decades of array signal processing research. IEEE signal processing magazine

Liu X, Katz J (2006) Instantaneous pressure and material acceleration measurements using a four-exposure PIV system. Exp Fluids 41:227-240

Lorenzoni V, Tuinstra M, Moore P, Scarano F (2009) Aeroacoustic analysis of a rod-airfoil flow by means of time-resolved PIV. In: 15th AIAA/CEAS aeroacoustics conference, Miami, Florida

Martinez-Lera P, Mueller A, Schram C, Rambaud P, Desmet W, Anthoine J (2008) Robust aeroacoustic computations based on curle's and powell's analogies. In: Proceedings of the international conference on noise and vibration engineering, pp 513-524

Moehring W (1978) On vortex sound at low mach number. J Fluid Mech 85:685

Scarano F, Riethmuller M (1999) Iterative multigrid approach in piv image processing with discrete window offset. Exp Fluids 26(6):513-523

Schram C, Tournour M, Martinez P (2007) Two dimensional in-duct vortex leapfrogging as a validation benchmark for internal aeroacoustics. In: Proceedings of academic science Philadelphia 13th international congress of sound and vibration 224, pp 449-463

Schröder A, Dierksheide U, Wolf J, Kompenhans J (2004) Investigation on trailing-edge noise sources by means of high-speed PIV. In: 12th International symposium on applications of laser techniques to fluid mechanics, Lisbon, Portugal

Siller H, Jacob M, Michel U (2005) Flow and noise modification by suction and blowing on a rod-airfoil configuration. Theor Comput Fluid Dyn 19:171-196

Tennekes H (1975) Eulerian and lagrangian time microscales in isotropic turbulence. J Fluid Mech 67(3):561-567

Violato D, Moore P, Scarano F (2009) Rod-airfoil flow investigation by time-resolved TOMO PIV for aeroacoustics. In: 8th International symposium on particle image velocimetry, Melbourne, Australia

Violato D, Moore P, Scarano F (2010) Three-dimensional timeresolved analysis of rod-airfoil flow for aeroacoustics. Submitted to Experiments in Fluids

Wang M, Freund J, Lele S (2006) Computational prediction of flowgenerated sound. Annual Rev Fluid Mech 18:483-512

Wernet M (2007) Temporally resolved PIV for space-time correlations in both hot and cold jet flows. Meas Sci Technol 18:1387-1403 